\title{
SORBÍMETRO \\ (Um aparêlho para a determinação das relações solo/água)
}

\author{
G. RANZANI e A. PORTA
}

Secção Técnica "Química Agrícola"

E. S. A. "Luiz de Queiroz" U. S. P. - Piracicaba

\section{INDICE}

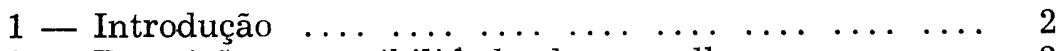

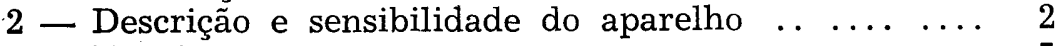

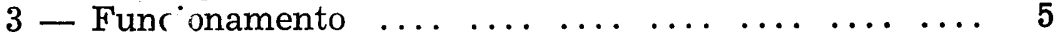

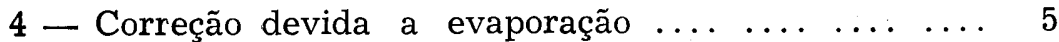

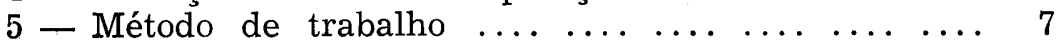

6 - Resultados experimentais e discussão $\ldots \ldots \ldots . . .10$

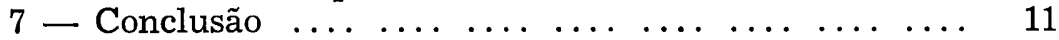

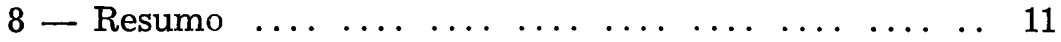

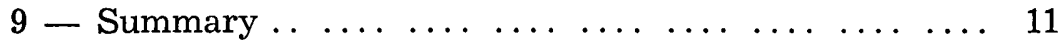

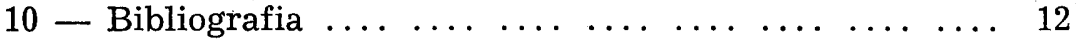




\section{INTRODUÇÃO}

As grandezas relacionadas ao dinamismo da água no solo, a ascensão capilar, a velocidade de ascensão, a absorção de água, são obtidas entre nós, utilizando amostras de terra fina sêca ao ar, (1), (2). A terra é colocada em tubos de vidro e posta em contato com um suprimento de água mantido à nível constante e os dados são obtidos pelo método gravimétrico.

As pesagens do tubo fornecem os elementos necessários aos cálculos da quantidade de água em função do tempo, para o que geralmente são empregadas, balanças com precisão de $1 \mathrm{~g}(2)$.

Com a instalação do sorbímetro como mostra a figura 1, é possível substituir o método ponderal pelo volumétrico, o que não apenas concorre para maior facilidade de manipulação, como torna o trabalho mais rigoroso. As leituras na bureta, podem ser feitas com precisão de $0,08 \mathrm{ml}$ (volume de ar carreado pela bolha).

\section{DESCRIÇÃO E SENSIBILIDADE DO APARELHO}

O sorbímetro (fig. 1), consta de uma bureta de $100 \mathrm{ml}$ cuja extremidade inferior abre-se no nível da água contida no vaso cônico $B$. O vaso $B$ liga-se ao tubo que contém a coluna de terra por um ramo horizontal que recebe água do depósito $D$ pela torneira $E$, e tem drenagem pela torneira $I$. A coluna de água da bureta dá uma variação de volume equivalente à sorção de água pela terra em $C$. A amostra de terra é acondicionada em tubos do tipo comum de percoladores, com $100 \mathrm{~cm}$ de comprimento e 2 a $3 \mathrm{~cm}$ de diâmetro; é ligado na extremidade $C$ do vaso cônico $B$ por conexão de borracha. A placa perfurada do tubo percolador e a extremidade inferior da bureta no vaso $B$ devem estar no mesmo plano horizontal. $O$ tubo com terra é sustentado por braçadeiras em um suporte de madeira, e acompanhado de uma régua milimetrada, destinada às leituras de coluna de terra e água absorvida.

A fim de possibilitar o estudo comparativo entre os métodos gravimétrico e volumétrico, as amostras de terra foram acondicionadas em tubos comuns de $2,4 \mathrm{~cm}$ de diâmetro e em $C$ foi adatado um pequeno funil, como mostra a fig. $2 a$.

A sensibilidade de funcionamento e a precisão das leituras dependem da coluna líquida que a bureta sustenta e da relação entre os diâmetros internos do vaso $B,(d 1)$ e da bureta (d2). 


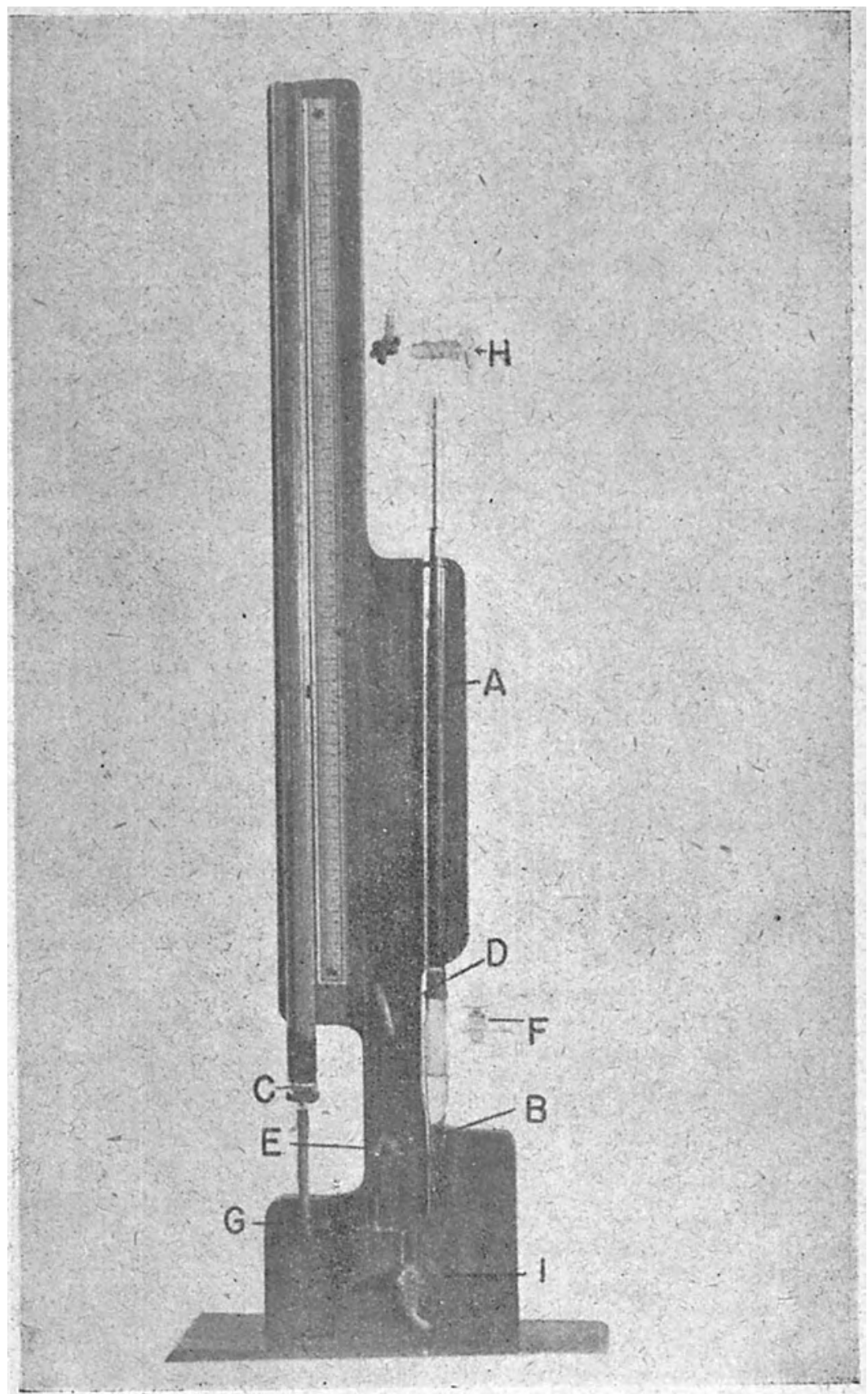

Fig. 1 - Sorbímetro 
Anais da E. S. A. "Luiz de Queiroz"

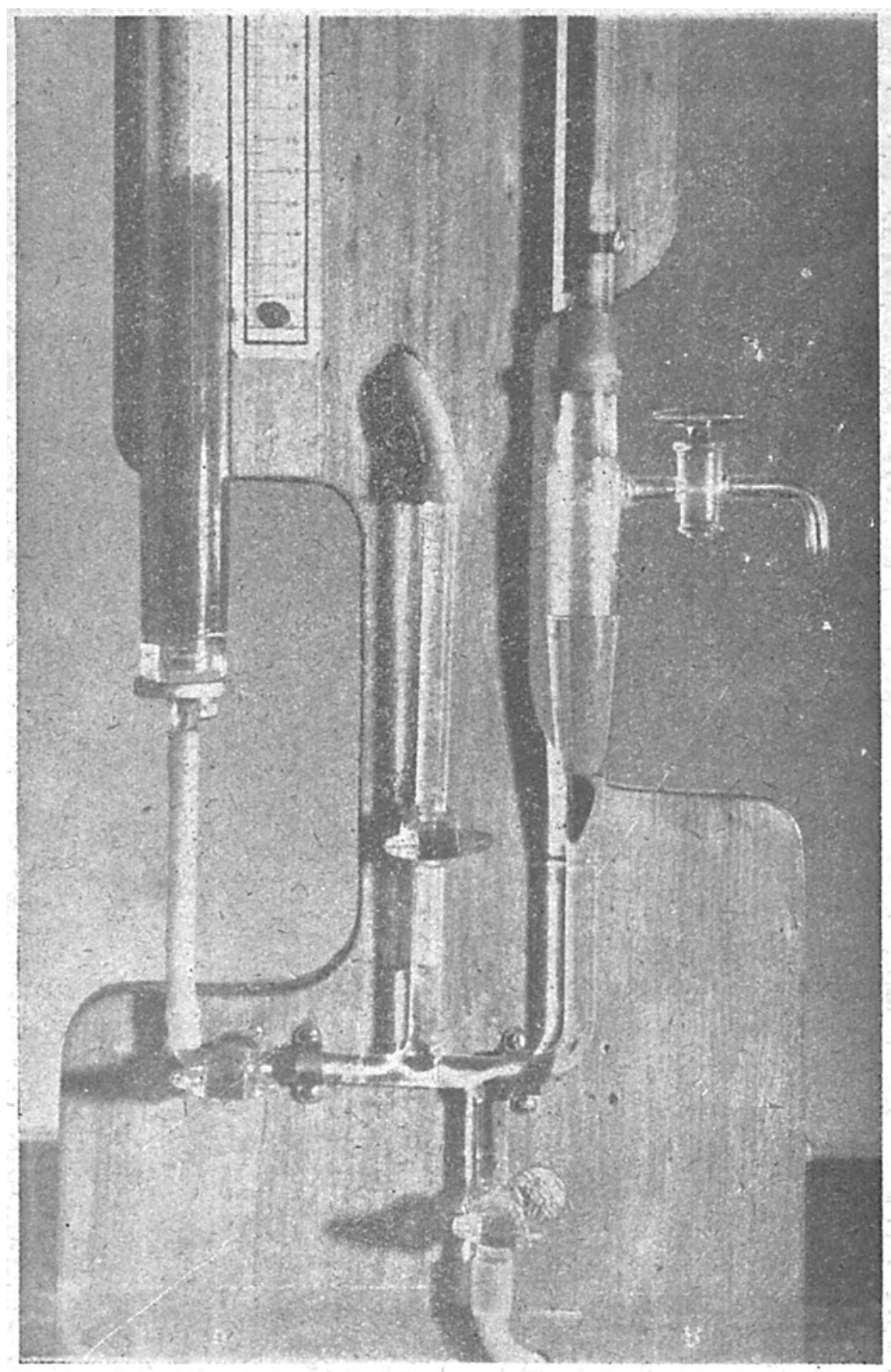

Fig. 1a. - Detalhe da ponte líquida 
No quadro 1 damos os valores obtidos experimentalmente, empregando uma bureta de $100 \mathrm{ml}$ cóm $1,35 \mathrm{~cm}$ de diâmetro interno, e fazendo leituras em 3 regiões da mesma de $0-20$, de $30-50$ e de $60-80 \mathrm{ml}$.

A expansão da bolha de ar, para igual coluna de água na bureta, aumenta com o valor da relação d1/d2. Quando o vaso $B$ oferece à abertura inferior da bureta uma secção com $1,55 \mathrm{~cm}$ de diâmetro, e a relação d1/d2 é de 1,15 , as leituras em qualquer ponto da bureta não variam apreciàvelmente.

A relação 1,15 foi por nós julgada conveniente à montagem do sorbímetro (fig. 1) e a sensibilidade dêste corresponde à $0,08 \mathrm{ml}$ (volume de ar carreado por uma bôlha ascendente), valor êste, bastante satisfatório.

\section{FUNCIONAMENTO}

Fechando-se as torneiras $G$ e $F$ e abrindo-se $H$ à pressão exterior, carrega-se a bureta por meio de $E$. Atingida a altura pouco superior à do traço inicial, fechar $E$ e abrir $F$ e $G$, o que restabelece a pressão exterior em $B$. Drenando-se o excesso de água por meio de $I$ até que o nível da água dos vasos coincida com a extremidade inferior da bureta, tem-se o conjunto pronto para receber a coluna de terra e iniciar as determinações.

\section{CORREÇÃO DEVIDA À EVAPORAÇÃO •}

Uma correção a ser feita é a da água evaporada pelas superfícies de exposição do conjunto. E' esta conseguida, instalando-se um evaporímetro (fig. $2 \mathrm{~b}$ ) que, em princípio, é semelhante ao sorbímetro por nós descrito.

A relação entre a superfície total exposta $S$ no sorbímetro e a superfície exposta $s$ no evaporímetro, é o fator de correção das leituras de volume no evaporímetro. Assim, a evapora. ção sendo representada por $E$, temos:

$$
\mathrm{E}=\frac{\mathrm{S}}{\mathrm{s}} \cdot \mathrm{v}
$$

em que $\mathrm{E}=$ evaporação no sorbímetro

$\mathrm{S}=$ superf. de evaporação no sorbímetro

$\mathrm{s}=$ superf. de evaporação no evaporímetro

$\mathrm{v}=$ leitura de volume no evaporímetro 


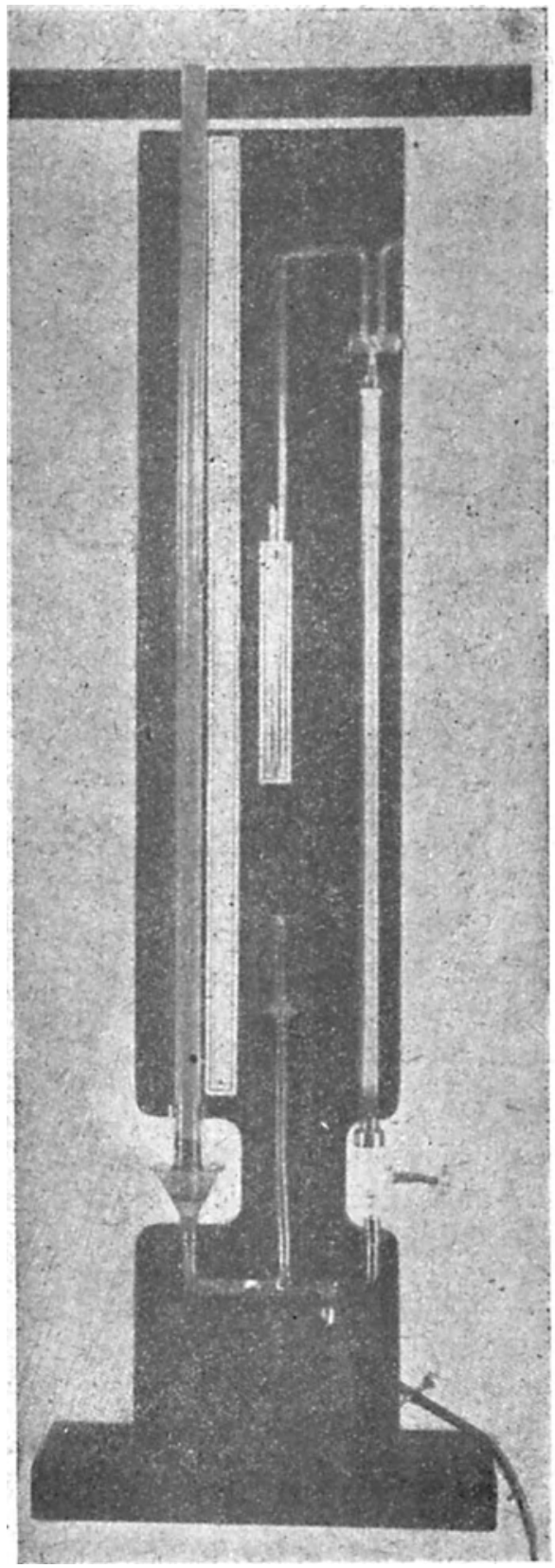

a

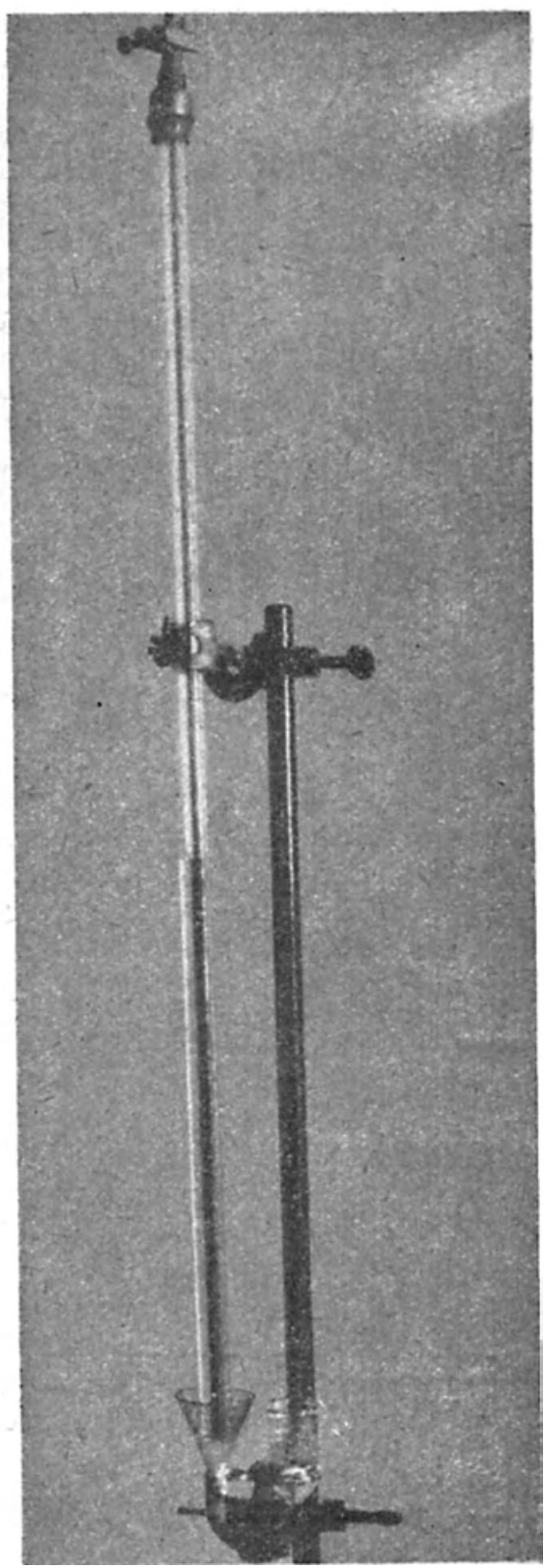

b

Fig. 2 - (a) Sorbímetro com funil adatado em (C), para obtenção de resultados com os métodos gravimétrico e volumétrico. (b) Evaporímetro utilizado para as correções 


\section{MÉTODO DE TRABALHO}

O tubo de vidro com $d \mathrm{~cm}$ de diâmetro munido de capuz metálico perfurado é carregado com $300-500 \mathrm{~g}$ de terra fina sêca ao ar. Depois de compactar convenientemente, é colocado no sorbímetro como mostra a fig. 2a.

Quando é utilizada a equação hiperbólica de VAGELER nos cálculos dos valores finais, temos que anotar:

$$
\begin{aligned}
& \text { hs = altura da coluna de terra no tubo } \\
& \text { Vo = volume inicial da bureta no sorbímetro } \\
& \text { vo }=\text { volume inicial da bureta no evaporímetro } \\
& \text { dia e hora da montagem. }
\end{aligned}
$$

Decorridas 24 horas, são feitas as leituras :

h24 = ascensão capilar em 24 horas

V24 = absorção de água + evaporação em 24 horas

v24 = evaporação em 24 horas

Após 120 horas anotam-se:

h120 = ascensão capilar em 120 horas

V120 = absorção + evaporação em 120 horas

V120 = evaporação em 120 horas.

Êstes dados nos permitem os cálculos da ascensão capilar máxima, velocidade de ascensão, absorção de água, velocidade de absorção e água capilar máxima do solo.

Calcula-se primeiro o valor $E$ correspondente à evaporação em 24 horas :

$$
\mathrm{E} 24=\frac{\mathrm{S}}{\mathrm{s}} \cdot(\mathrm{v} 24-\mathrm{vo})
$$

e a 120 horas :

$$
\mathrm{E} 120=\frac{\mathrm{S}}{\mathrm{s}}(\mathrm{v} 120-\mathrm{vo})
$$

Em seguida corrigem-se as leituras no sorbímetro obtendose os valores de:

5.1. Água absorvida pelo solo:

Água absorvida em 24 horas, A24 = V24 - $(\mathrm{E} 24+\mathrm{Vo})$ Água absorvida em 120 horas, $\mathrm{A} 120=\mathrm{V} 120-(\mathrm{E} 120+\mathrm{Vo})$ 


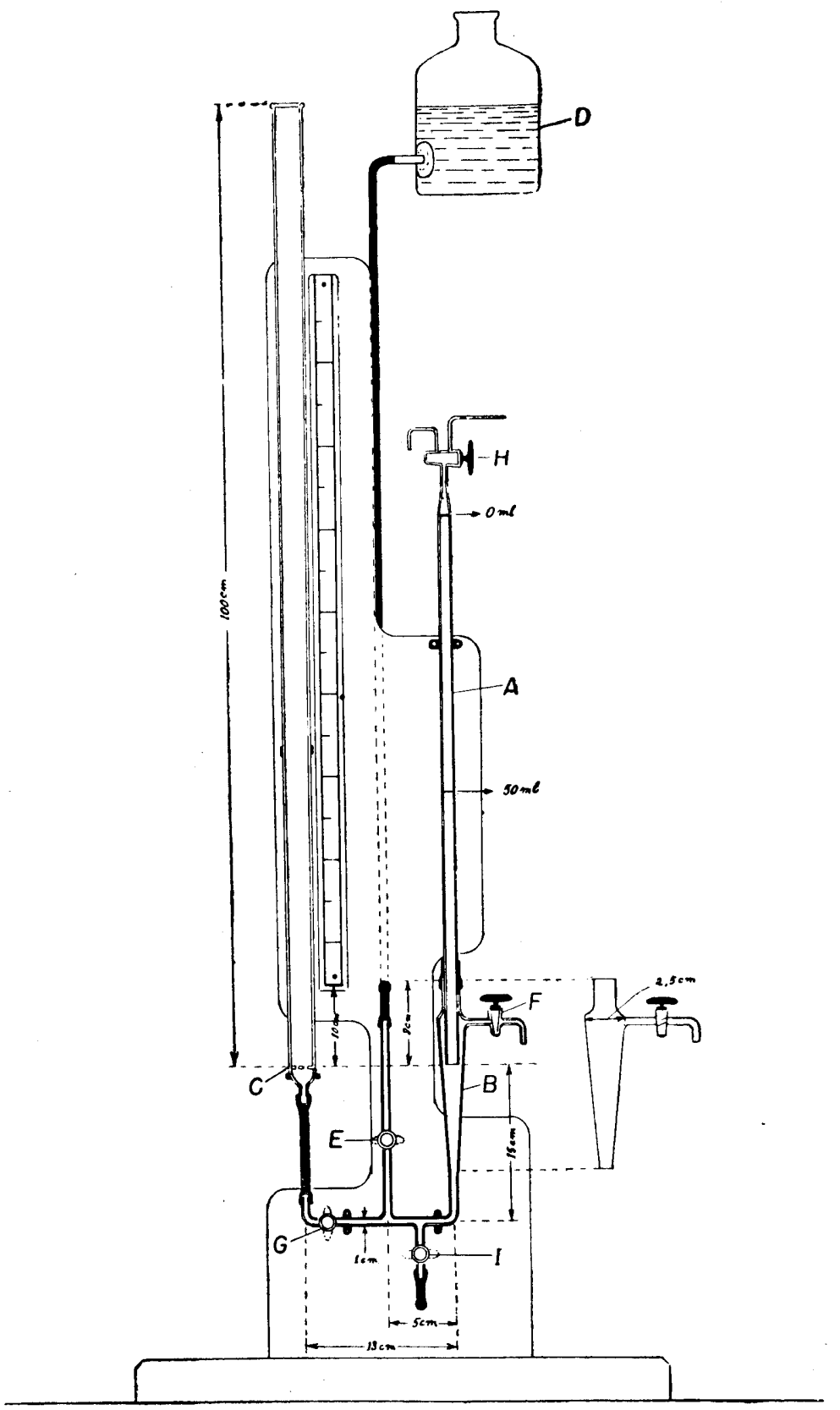

Fig. 3 - Esquema do aparelho 
5.2. Ascensão capilar máxima (hT) em $\mathrm{mm}$ :

$$
\mathrm{hT} \frac{4 \mathrm{~h} 24 \ldots \mathrm{h} 120}{5 \mathrm{~h} 24-\mathrm{h} 120}
$$

5.3. Velocidade máxima de ascensão capilar (Vh) em mm/hora.

$$
\mathrm{Vh}=\frac{\mathrm{h} 24 . \mathrm{h} 120}{30(\mathrm{~h} 120-\mathrm{h} 24)}
$$

5.4. Altura máxima de água absorvida (HT) em mm :

Altura absorvida em 24 horas, $\mathrm{H} 24=\frac{40}{\pi \mathrm{d} 2} \cdot$ A24 $(\mathrm{mm})$.

Altura absorvida em 120 horas, $\mathrm{H} 120=\frac{40}{\pi \mathrm{d} 2}$. A120 (mm). em que $d=$ diâmetro do tubo que contém a amostra de terra.

A altura máxima de água absorvida, referida ao volume do tubo com diâmetro de $2,4 \mathrm{~cm}$ é :

$\mathrm{H}=0,222 \mathrm{~V}$ em $\mathrm{cm} \quad \mathrm{ou}$,

$\mathrm{H}=2,22 \mathrm{~V} \mathrm{~mm}$, e, as alturas de água absorvida em $24 \mathrm{e}$ 120 horas são, respectivamente :

$\mathrm{H} 24=2,22 \mathrm{~A} 24$

$\mathrm{H} 120=2,22 \mathrm{~A} 120$

A altura máxima de água absorvida é :

$$
\mathrm{HT}=\frac{4 \mathrm{H} 24 \cdot \mathrm{H} 120}{5 \mathrm{H} 24-\mathrm{H} 120}
$$


5.5. Velocidade máxima de absorção (VH) em $\mathrm{mm} / \mathrm{hora:}$

$$
\mathrm{VH}=\frac{\mathrm{H} 24 \cdot \mathrm{H} 120}{30(\mathrm{H} 120-\mathrm{H} 24)}
$$

5.6. Água capilar máxima (A\%) em \% de terra fina sêca ao ar

Êste valor, segundo PAIVA NETO e DE JORGE (2) identifica-se com a quantidade de água absorvida por $100 \mathrm{~g}$ de T. F. S. A. em 120 horas.

$$
\mathrm{A} \%=\frac{100 \mathrm{hs}}{\mathrm{X} \cdot \mathrm{h} 120} \cdot \mathrm{A} 120
$$

quando utilizamos $X$ g de terra e, hs e h120 representam respectivamente a altura da coluna de solo no tubo e a altura da coluna capilar em 120 horas.

\section{RESULTADOS EXPERIMENTAIS E DISCUSSÃO}

Os resultados obtidos com os métodos gravimétrico (1) e volumétrico por nós proposto, referem-se a terras do município de Piracicaba e estão contidos no quadro 2. Empregamos tubos de vidro com diâmetro interno de $2,4 \mathrm{~cm}$.

Os sorbímetros utilizados apresentaram uma relação $\mathrm{d} 1 / \mathrm{d} 2=1,58$ e foram montados como mostra a fig. 2

Os valores de $A$ pelo método gravimétrico, referem-se às diferenças $(\mathrm{P} 24-\mathrm{P})$ e $(\mathrm{P} 120-\mathrm{P})$ com um êrro de $\pm 1 \mathrm{~g}$. (precisão da balança); pelo método sorbimétrico proposto, representam as leituras V24 e V120 corrigidas, ou ainda:

$$
\begin{aligned}
\mathrm{A} 24 & =\mathrm{V} 24-(\mathrm{E} 24+\mathrm{Vo}) \\
\mathrm{A} 120 & =\mathrm{V} 120-(\mathrm{E} 120+\mathrm{Vo})
\end{aligned}
$$

O evaporímetro apresentou uma área exposta de $5,4 \mathrm{~cm}^{2}$ e a relação $\frac{\mathrm{S}}{\mathrm{S}}=1,2$, êste último constituindo, como vimos, o fator da evaporação a corrigir nas leituras sorbimétricas.

Os resultados de A24, A120 e A\% obtidos pelos dois métodos, não diferem entre si, como revelado pelo teste " $t$ ". 
Assim :

$$
\begin{aligned}
& \mathrm{A} 24 \ldots \ldots \ldots \ldots \mathrm{t}=\frac{49,17-48,81}{5,36}=0,067 \\
& \mathrm{~A} 120 \ldots \ldots \ldots \mathrm{t}=\frac{59,47-57,70}{6,32}=0,28 \\
& \mathrm{~A} \% \ldots \ldots \ldots \mathrm{t}=\frac{27,90-26,93}{1,45}=0,67
\end{aligned}
$$

\section{CONCLUSÃO}

$\mathrm{O}$ sorbímetro apresentando uma relação $\mathrm{d} 1 / \mathrm{d} 2=1,58$ reproduz com suficiente exatidâo os resultados obtidos com o método gravimétrico. Sôbre êste método, apresenta a vantagem da obtenção de resultados em espaços de tempo curtos, sem importar em possíveis distúrbios ou interrupção do suprimento de água à base da coluna de solo. Além das determinações aqui discutidas, presta-se à obtenção do coeficiente de absorção capilar e da condutividade hidráulica ou constante $\mathrm{K}$ de Darcy, como teremos oportunidade de expôr em outro trabalho.

\section{RESUMO}

Os autores descrevem um aparelho destinado à medida contínua da água absorvida pela TFSA em condições de laboratório.

O sorbímetro, cujo funcionamento obedece ao princípio dos vasos comunicantes, consta de um tubo em U cujos ramos verticaìs se ligam respectivamente à uma coluna de terra e à uma bureta de $100 \mathrm{ml}$. A bureta restabelece no tubo, a água absorvida pela terra. As leituras de bureta substituem as pesagens do método gravimétrico.

$\mathrm{O}$ aparelho é recomendado para medidas a intervalos de tempo para os quais, o método usual é ineficiente.

\section{SUMMARY}

This paper describes an apparatus designed to carry on the continuous measurement of the water absorption by soils under laboratory conditions. The apparatus was called "sorbimetro". 
The principle accordingly to the "sorbimetro" works is the same which governs the flow of water in the usual linked flasks. The apparatus has a $U$ tube whose vertical arms are connected respectivelly, to a soil colunn and to a $100 \mathrm{ml}$ burete; the burete keeps constant the volume of water in the $U$ tube since the liquid is being absorbed by the soil. In the present method the readings on the burete substitute for the usual weighings. The method is recommended for measurements in time intervals in which the gravimetric method does not work too well.

\section{BIBLIOGRAFIA}

1 - VAGELER, P. e T. DE CAMARGO, 1936 - "Analyses de solos. 1 - Analyse Physica”, Inst. Agron. de Campinas, Boletim técnico n. 24.

2 - PAIVA NETTO, J. E. e W. DE JORGE, 1947 - Estudo preliminar do sistema água-solo-planta no Estado de S. Paulo. Bragantia 7: 133-150.

\section{QUADRO 1}

Variação das leituras em três regiões da bureta : 0 a 20,30 a 50 e 60 a 80

\begin{tabular}{c|c|c|c}
\hline $\mathrm{d} 1 / \mathrm{d} 2$ & $\begin{array}{c}\text { Região de } \\
\text { leitura na } \\
\text { bureta }\end{array}$ & $\begin{array}{c}\text { Variação de } \\
\text { volume por } \\
\text { bolha }\end{array}$ & $\begin{array}{c}\text { Variação } \\
\text { relativa } \\
(*)\end{array}$ \\
\hline 3,0 & $0-20$ & 1,50 & 0,30 \\
3,0 & $30-50$ & 1,30 & 0,10 \\
3,0 & $60-80$ & 1,20 & 0,00 \\
2,0 & $0-20$ & 1,30 & 0,10 \\
2.0 & $30-50$ & 1,20 & 0,00 \\
2,0 & $60-80$ & 1.20 & 0,00 \\
1,5 & $0-20$ & 0,30 & 0,05 \\
1,5 & $30-50$ & 0,25 & 0,00 \\
1,5 & $60-80$ & 0,25 & 0,00 \\
1,2 & $0-20$ & 0,17 & 0,02 \\
1,2 & $30-50$ & 0,16 & 0,01 \\
1,2 & $60-80$ & 0,15 & 0,00 \\
1,15 & $0-20$ & 0,08 & 0,00 \\
1,15 & $30-50$ & 0,08 & 0,00 \\
1,15 & $60-80$ & 0,08 & 0,00 \\
\hline
\end{tabular}

(*) referida à leitura $60-80$. 
QUADRO 2

Comparação dos métodos gravimétrico e volumétrico

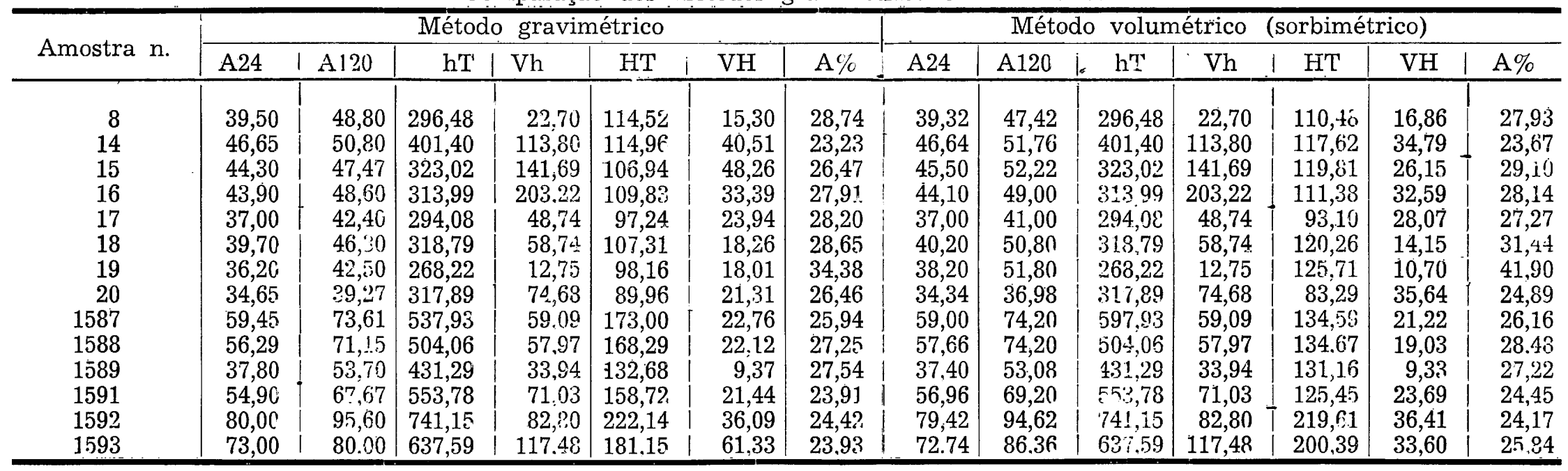

A24 ... . absorção de água em 24 horas

A120....absorção de água em 120 horas

hT ....ascensão capilar máxima, em $\mathrm{mm}$

Vh ....velocidade máxima de ascensão capilar, em $\mathrm{mm}$ / hora
HT ....altura máxima de água absorvida, em $\mathrm{mm}$

VH ... velocidade máxima de absorção, em $\mathrm{mm} /$ hora

A\% .... água capilar máxima, p/ $100 \mathrm{~g}$ de terra fina sêca ao ar. 